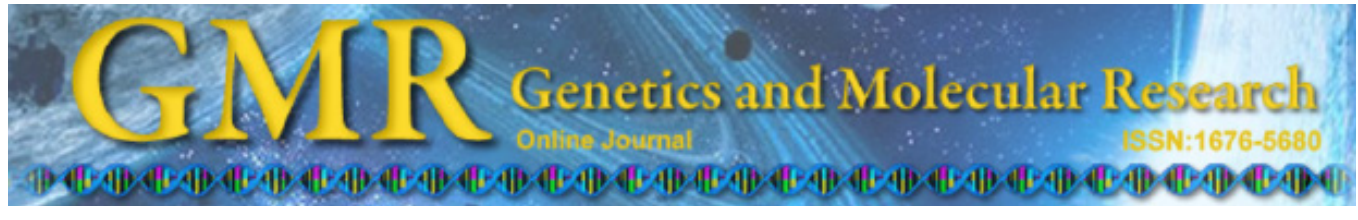

\title{
Molecular cloning of HSP70 in Mycoplasma ovipneumoniae and comparison with that of other mycoplasmas
}

\author{
M. Li ${ }^{1,2}$, C.J. Ma ${ }^{1,2,3}$, X.M. Liu ${ }^{1,2}$, D. Zhao ${ }^{1,2}$, Q.C. Xu ${ }^{1,2}$ and Y.J. Wang ${ }^{1,2}$ \\ ${ }^{1}$ Key Laboratory of Ministry of Education for Conservation and Utilization of \\ Special Biological Resources of Western China, Yinchuan, Ningxia, China \\ ${ }^{2}$ College of Life Science, Ningxia University, Yinchuan, Ningxia, China \\ ${ }^{3}$ Key Laboratory of Ministry of Education for Fertility Preservation and \\ Maintenances, Ningxia Medical University, Yinchuan, Ningxia, China \\ Corresponding author: Y.J. Wang \\ E-mail: wyj@nxu.edu.cn / yujiongw@gmail.com
}

Genet. Mol. Res. 10 (2): 834-848 (2011)

Received December 6, 2010

Accepted February 21, 2011

Published May 10, 2011

DOI 10.4238/vol10-2gmr1193

\begin{abstract}
Mycoplasma ovipneumoniae, a bacterial species that specifically affects ovine and goat, is the cause of ovine infectious pleuropneumonia. We cloned, sequenced and analyzed heat shock protein 70 (HSP70) (dnaK) gene of $M$. ovipneumoniae. The full length open reading frame of the $M$. ovipneumoniae HSP70 gene consists of 1812 nucleotides, with a $\mathrm{G}+\mathrm{C}$ content of $34.16 \%$, encoding 604 amino acids. Comparative analysis with the HSP70 sequences of 15 Mycoplasma species revealed 59 to $87 \%$ DNA sequence identity, with an amino acid sequence identity range of 58 to $94 \%$. M. ovipneumoniae and $M$. hyopneumoniae shared the highest DNA and amino acid sequence identity ( 87 and 94\%, respectively). Based on phylogenetic analysis, both the DNA and amino acid identities of $M$. ovipneumoniae with other mycoplasmal HSP70 were correlated with the degree of relationship between the species.
\end{abstract}


The C-terminus of the HSP70 was cloned into a bacterial expression vector and expressed in Escherichia coli cells. The recombinant C-terminal portion of HSP70 protein strongly reacted with convalescent sera from M. ovipneumoniae-infected sheep, based on an immunoblotting assay. This indicates that HSP70 is immunogenic in a natural $M$. ovipneumoniae infection and may be a relevant antigen for vaccine development.

Key words: Mycoplasma ovipneumoniae; Heat shock protein 70; HSP70; Immune response; Recombinant protein; Bioinformatic analysis

\section{INTRODUCTION}

Mycoplasmas, the smallest and simplest self-replicating organisms, lack a cell wall and contain the minimal complement of life enabling genes (Razin et al., 1998). Many species of mycoplasmas are known pathogens of man and other mammals, including M. genitalium, M. pneumoniae and M. hyopneumoniae (M. hyo). Despite the genome and cellular structure simplicities, diseases caused by mycoplasma infection are complex. To date, relatively less attention has been paid to mycoplasmal diseases. Genomic sequencing of mycoplasmas, including M. genitalium (Fraser et al., 1995), M. pneumoniae (Himmelreich et al., 1996) and M. hyo (Minion et al., 2004), has led to a better understanding of the entire machinery of a self-replicating cell and mycoplasma pathogenesis. Pathogenic mycoplasma infections in mammals are usually chronic in nature. The host immune and inflammatory responses induced by mycoplasma infections are more suggestive of damage rather than the direct effects of mycoplasmal cell virulent components (Biberfeld, 1985). Mycoplasma infection is able to induce specific and nonspecific immune reactions that modulate host immune responsiveness contributing to their pathogenic properties. The properties of immunomodulation of mycoplasmas suppress and evade the host defense mechanisms, leading to chronic and persistent infection (Biberfeld, 1985).

The heat-shock proteins (HSPs) are a group of proteins induced by environmental stress conditions, which play an important role in stimulating both host innate and adaptive immunities (Craig, 1985; Torigoe et al., 2009). HSPs can be classified into six families by their molecular weight: large molecular weight HSP family, HSP90 family, HSP70 family, HSP60 family, small molecular weight HSP family, and ubiquitin (Craig et al., 1993). HSP60 and HSP70 families are the most conserved and abundant (Craig, 1985; Craig et al., 1993). HSP70 has been extensively studied and is known to function as a molecular chaperone, anti-cell apoptosis agent, antioxidant, inducing immune responses, improving stress tolerance, cell proliferation promotion, cytoskeleton formation, and repair (Kiang and Tsokos, 1998). Both HSP70 and HSP60 are immunodominant antigens and pathogens in bacteria and mycoplasmas. They have been shown to induce immune responses protecting hosts against bacterial and mycoplasmal infections (Scherm et al., 2002; Floto et al., 2006; Amemiya et al., 2007; Rasoli et al., 2010). Previous bioinformatic analysis of $M$. hyo and the other nine mycoplasmas whose genomes have been sequenced has suggested that HSP70 
(DnaK) with the downstream DnaJ and Grp formed a chaperone protein complex (DnaKDnaJ-GrpE). The N-terminal domain of the HSP70 protein is important in its chaperone function. The C-terminal portion is featured as the immunodominant antigen and functions as an immune adjuvant that induces and/or enhances the host immune responses (Kakeya et al., 1999). Of note, the HSP60 (GroEL) is absent in the strain of M. hyo (Barré et al., 2004; Minion et al., 2004).

Mycoplasma ovipneumoniae is a species of Mycoplasma bacteria that specifically infects ovine. M. ovipneumoniae is the infectious agent in ovine pleuropneumonia causing lethal pneumonia in sheep and goats (Staint George and Carmichael, 1975; Foggie et al., 1976; Ionas et al., 1991; Lin et al., 2008; Dassanayake et al., 2010). This organism is highly infectious and is prevalent in almost every flock, resulting in major economic losses worldwide in the ovine industry. Compared to other pathogenic mycoplasmas, studies on $M$. ovipneumoniae are limited by many aspects including the lack of the entire genomic sequence. This substantially hinders the understanding of the molecular basis and pathogenic mechanisms of M. ovipneumoniae infection. Both M. ovipneumoniae and M. hyopneumoniae are members of the order Mycoplasmales. Bioinformatic analysis of M. ovipneumoniae known genomic sequences, also revealed that M. ovipneumoniae and M. hyopneumoniae share high homology, suggesting that the two species of Mycoplasma may exhibit similar mechanisms of active phenotypic switch and antigenic variation (Minion et al., 2004). It has been shown that M. hyopneumoniae lacks the HSP60 (GroEL) gene and that monoclonal antibodies generated against part of $M$. hyo HSP70 with sequence homologies to HSP70 of M. genitalium and Bacillus subtilis were capable of blocking the growth of Mhp (Chou et al., 1997). This could imply that $M$. ovipneumoniae HSP70 may be used as a vaccine candidate to induce host immune responses against $M$. ovipneumoniae infection.

The M. ovipneumoniae HSP70 gene was cloned and characterized in the present study to better understand the potential immunogenic function of HSP70 of M. ovipneumoniae against mycoplasma infection in ovine. The immune responses against HSP70 in $M$. ovipneumoniae-infected animals were also evaluated by immunoblotting using sheep convalescent sera.

\section{MATERIAL AND METHODS}

\section{Plasmids, cell lines and bacterial strains}

M. ovipneumoniae Queensland Strain Y98 (Jones et al., 1976) was purchased from the China Institute of Veterinary Drug Control (Beijing, China). The mycoplasma bacterial strain was cultured in the media described previously (Jones et al., 1976). Escherichia coli competent cells JM109 and BL21 (DE3) were used to produce recombinant plasmids and recombinant proteins. E. coli strains were grown in LB medium supplemented with ampicillin. Bacterial expressing plasmid pET-28a was used for generation of recombinant proteins (Novagen, USA).

\section{Reagents}

Restriction endonuclease and DNA modifying enzymes were products from Takara 
Biologicals (Japan), New England Biobabs (USA) or Promega (USA). Chemicals used in this study were products from Sigma (USA). Bacterial genomic DNA isolation kit, Plasmid miniprep kit, reverse transcriptase polymerase chain reaction (RT-PCR) kit, TA Clone kit, DNA ladder, Pre-stained protein marker, Mouse Anti-6X His antibody, and HRP-goat anti-IgG were purchased from TianQen Biological Inc. (China). The M. ovipneumoniae detection ELISA kit (M. ovipneumoniae Queensland Strain Y98 was used as antigen component in the kit) was certified by the China Institute of Veterinary Drug Control, and manufactured by the Lanzhou Institute of Biological Products (Lanzhou, China).

\section{M. ovipneumoniae HSP70 DNA cloning}

M. ovipneumoniae bacterial genomic DNA was isolated using a bacterial genomic isolation kit and used as a template for PCR cloning of the HSP70 DNA fragments. To amplify, clone and sequence HSP70 DNA of M. ovipneumoniae, the experimental procedure comprised five sequential steps. Primers used in this procedure are listed in Table 1. Degenerate primers were designed based on the conserved amino acid and DNA sequences of 15 species of Mycoplasma HSP70. Step 1: Seven degenerate primers (three forward and four reverse with 12 pairs of primer combinations) were used to perform 12 individual PCRs for amplification of HSP70 DNA fragment 1 (F1 in Table 1). PCRs were carried out using a Touchdown (TD) PCR program for 20 cycles $\left(45 \mathrm{~s}\right.$ at $95^{\circ} \mathrm{C}, 30$ $\mathrm{s}$ at $60^{\circ} \mathrm{C}, 60 \mathrm{~s}$ at $72^{\circ} \mathrm{C}$, followed by a $0.5^{\circ} \mathrm{C}$ decrease in the annealing temperature every cycle). After completion of the TD program, 15 cycles were subsequently performed $\left(95^{\circ} \mathrm{C}\right.$ for $30 \mathrm{~s}, 50^{\circ} \mathrm{C}$ for $30 \mathrm{~s}$ and $72^{\circ} \mathrm{C}$ for $\left.60 \mathrm{~s}\right)$ ending with a 5 -min extension at $72^{\circ} \mathrm{C}$. The resulting PCR products were cloned into a pMD18-T vector (Takara Biologicals, Japan) and sequenced. The clones harboring an F1 of $M$. ovipneumoniae HSP70 DNA fragment were identified by homological analysis using the HSP70 sequences from other Mycoplasma species. Step 2: Using the F1 fragment sequence of the HSP70, three specific forward primers and one reverse degenerate primer (3 pairs of primer combinations) were employed in three PCRs to amplify HSP70 DNA fragment 2 (F2 in Table 1), using the TD PCR program identical to Step 1. The PCR products were cloned into the pMD18-T vector, sequenced and identified as described in Step 1. Step 3: Based on the sequence of the above F2 fragment of HSP70, a specific forward primer and two reverse degenerate DnaJ primers (2 pairs of primer combinations) were used for two PCRs to amplify a 3 '-terminal fragment (Table 1). This was done by using a TD PCR program for 10 cycles $\left(45 \mathrm{~s}\right.$ at $95^{\circ} \mathrm{C}, 30 \mathrm{~s}$ at $60^{\circ} \mathrm{C}, 60 \mathrm{~s}$ at $72^{\circ} \mathrm{C}$, followed by a $1.0^{\circ} \mathrm{C}$ decrease of the annealing temperature every cycle), followed by 20 cycles of PCR amplification $\left(95^{\circ} \mathrm{C}\right.$ for $30 \mathrm{~s}$, $50^{\circ} \mathrm{C}$ for $30 \mathrm{~s}$ and $72^{\circ} \mathrm{C}$ for $60 \mathrm{~s}$ ) ending with a 5 -min extension at $72^{\circ} \mathrm{C}$. PCR products were cloned, sequenced and identified as in Step 1. Step 4: The tail-PCR strategy was used for the 5'-terminal fragment of MO HSP70 DNA. An adaptor primer obtained from the genome walking kit was used as the forward primer and three reverse primers were designed based on fragment 1 of HSP70 DNA for nested PCR. The final PCR product was cloned, sequenced and identified as in Step 1. Step 5: The final HSP70 sequence was compiled from the above PCR fragment sequences using the Vector NTI 11 ContigExpress software. PCR amplification using P1 and P3 primers (Table 1) was used for amplification of the full length of M. ovipneumoniae HSP70 DNA. The PCR fragment 
Table 1. Primers used for the amplification and cloning of the Mycoplasma ovipneumoniae HSP70 gene.

\begin{tabular}{lllc}
\hline Fragment & Primer & Sequence $\left(5^{\prime} \rightarrow 3^{\prime}\right)$ & Degeneracy \\
\hline F1 & HS1A & Fwd: GAYYTWGGWACHACHAACTC & 144 \\
& HS1B & Fwd: GAYYTWGGWACHACHAATTC & 144 \\
& HS2 & Fwd: GGWACNTTTGAYGTHTC & 48 \\
& HA2 & Rev: GADACRTCAAANGTWCC & 48 \\
& HA3 & Rev: ACHACYTCRTCHGGRTT & 72 \\
& HA4A & Rev: GWWADHGGDGTWACATC & 216 \\
& HA4B & Rev: GWWADHGGDGTWACGTC & 216 \\
\hline F2 & HSS1 & Fwd: ATTGGTCACAAAGTTTCAAAAGCTGT & NA \\
& HSS2 & Fwd: GATAATGCTCAACGTGAAGCGACA & NA \\
& HSS3 & Fwd: GAACCAACAGCAGCCGCACTGACATT & NA \\
\hline 3'-terminus & HA4 & Rev: GWWADHGGDGTWACRTC & 432 \\
\hline HSS7 & Fwd: GCCAAATCGTTCAATAAATCCTGATG & NA \\
& DnaJ1 & Rev: RTCNGGRTGRTA & 32 \\
& DnaJ2 & Rev: ARDATYTCRTANGCYTC & 192 \\
\hline Full length & AP & Fwd: Adaptor Primer (Takara Genome Walking kit) & NA \\
& HSA1 & Rev: ACAGATGCGATTGCTTCAGGGTTAGT & NA \\
\hline C-terminal & HSA2 & Rev: ACAATTTCCTCACCATTTTGAAGGC & NA \\
cloning & HSA3 & Rev: ACAGGTTTTGATTTTCGATAATTGC & NA \\
\hline & P1 & Fwd: ATGAAAGGAAAACATAATATGGC & NA \\
P3A & P2 & Rev: GGGGTCGACTTAATTTTGTTTGATTTC & NA \\
\hline
\end{tabular}

$\mathrm{NA}=$ not available. The italic and underlined sequences indicate the restriction enzyme sites used for cloning (SalI and $\mathrm{BamHI}$ ).

was cloned into the pMD-18T vector to generate the pMD-MoHsp70 plasmid containing a full length of M. ovipneumoniae HSP70 DNA.

\section{Expression of the recombinant C-terminal portion of the M. ovipneumoniae HSP70}

The above pMD-MoHsp70 plasmid served as the template to amplify the 3'-terminus of the M. ovipneumoniae HSP70 gene using primers P2 and P3. The PCR fragment was cloned in frame into the pET-28a $(+)$ bacterial expression vector. After being modified by BamHI-SalI digestions, the resultant vector was designated as pET-MoHsp70C and used for expression of recombinant His-tag-HSP70C fusion protein in E. coli BL21 (DE3) cells according to the manufacturer instruction.

\section{Genetic analysis of the M. ovipneumoniae HSP70 gene}

The NCBI Open Reading Frame (ORF) Finder was utilized to identify the ORF of M. ovipneumoniae HSP70 for the above cloned DNA sequence (http://www.ncbi.nlm.nih. gov/gorf/gorf.html). Sequence alignments, translations, and comparisons were carried out using DNAMAN (v. 4.1, Lynnon Bio-Soft, Vaudreuil, Canada). The BLAST algorithm was used to search the NCBI GenBank (http://www.ncbi.nlm.nih.gov/) databases for HSP70 homologous sequences of the 15 known Mycoplasma species (strains). Phylogenetic trees of 
DNA and protein were constructed using the neighbor-joining method with the DNAMAN software; bootstrap values were calculated on 1000 replicates of the alignment (Saitou and Nei, 1987; Kumar et al., 2004). The DNA Star software was used to analyze the antigenicity and surface probability.

\section{Detection of anti-HSP70 antibodies in the convalescent sera of $M$. ovipneumoniae- infected sheep}

E. coli BL21 (DE3) cells expressing recombinant His-tag-HSP70C fusion protein were lysated in lysis buffer $(20 \mathrm{mM}$ Tris-Bis propane, $50 \mathrm{mM} \mathrm{NaCl}, 1 \mathrm{mM}$ DTT, $2 \mathrm{mM}$ EDTA, $2.5 \mu \mathrm{g} / \mathrm{mL}$ Lyzozyme, $\mathrm{pH}$ 8.5), sonicated and centrifuged. The protein concentration of the above supernatant was determined by a slightly modified Bradford method using known standards (Ramagli, 1999). The samples were electrophoretically separated on a 10\% SDS-PAGE, followed by Coomassie G250 blue silver staining to evaluate protein expression (Candiano et al., 2004). The nitro-cellulose membranes blotted with the $E$. coli lysate containing His-tag-HSP70C protein were used to determine the anti-mycoplasmal antibodies in the convalescent sera of sheep by Western blotting. The convalescent sera were collected from the M. ovipneumoniae-infected sheep, which were confirmed by ELISA. The primary M. ovipneumoniae HSP70 antibody in the convalescent sera was detected by horseradish peroxidase-coupled horse anti-sheep conjugate and visualized using DAB substrate.

\section{RESULTS}

\section{Cloning of the M. ovipneumoniae full-length HSP70 DNA}

Conservative homology alignments of the HSP70 gene were performed between 15 Mycoplasma species (strains) (Table 2). Degenerative primers were used to clone M. ovipneumoniae HSP70 DNA fragments step by step as illustrated in Figure 1A. The PCR fragments were cloned into the pMD-18T vector, sequenced and identified as fragments of the HSP70 gene by alignment of their nucleotide and predicted amino acid sequences to HSP70 sequences of M. hyopneumoniae. Specific PCR products were only obtained from reactions containing primers with a combination of HS1A and HA2 for fragment 1 (Step 1), and HSS7 and DnaJ1 for the 3'-terminus (Step 3) (Figure 1B). PCRs using the primer combinations in Steps 2, 4 and 5 produced the expected products (Figure 2B and data not shown). The PCR fragment sequences obtained from the series cloning steps above were assembled using the Vector NTI 11 ContigExpress software by comparing the HSP70 sequences of the other Mycoplasma species (strains) (Figure 2). The resulting sequence was identical to the sequence of the PCR product amplified from the M. ovipneumoniae genomic DNA using the P1 and P3 primers. The PCR product of the full-length HSP70 gene was cloned into the pMD-18T vector to generate the pMD-MoHsp70 vector. The identified full-length HSP70 gene was highly conserved between the other Mycoplasma species (strains), suggesting successful cloning of $M$. ovipneumoniae HSP70. 


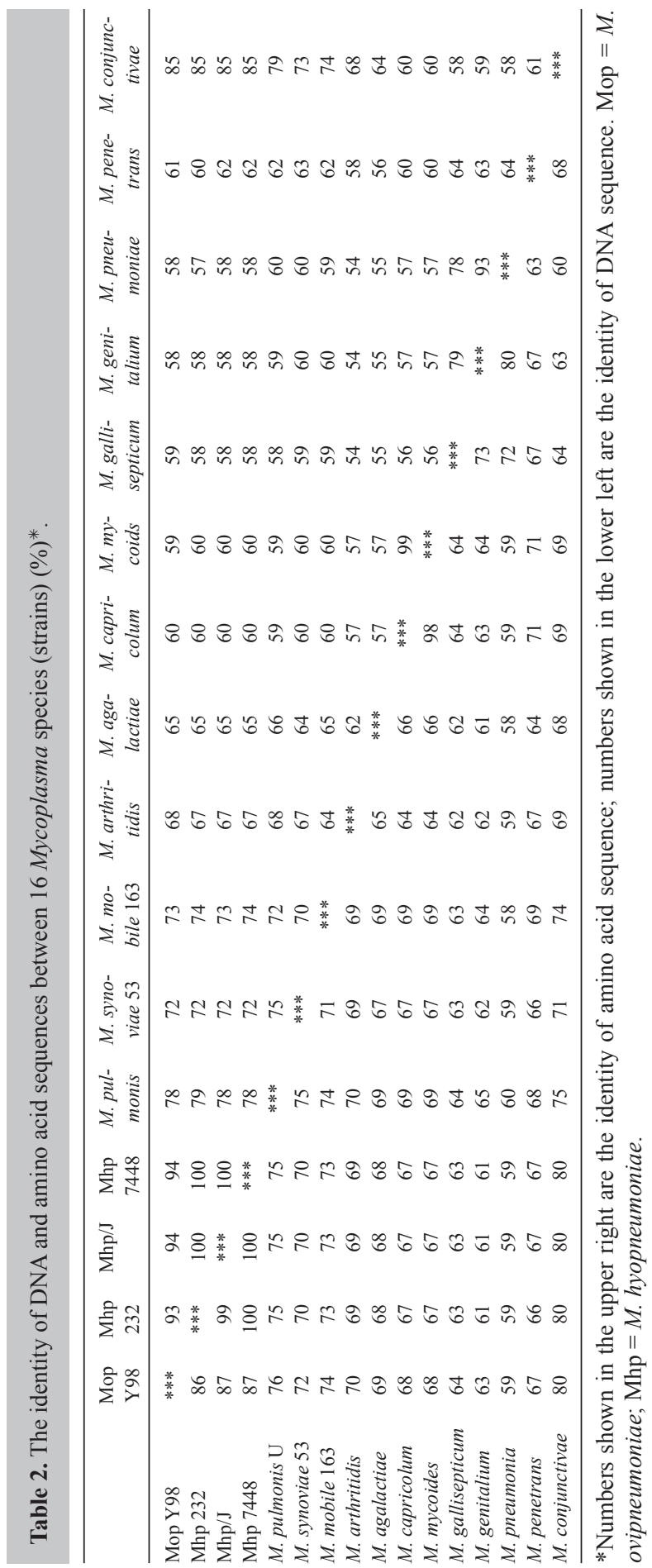




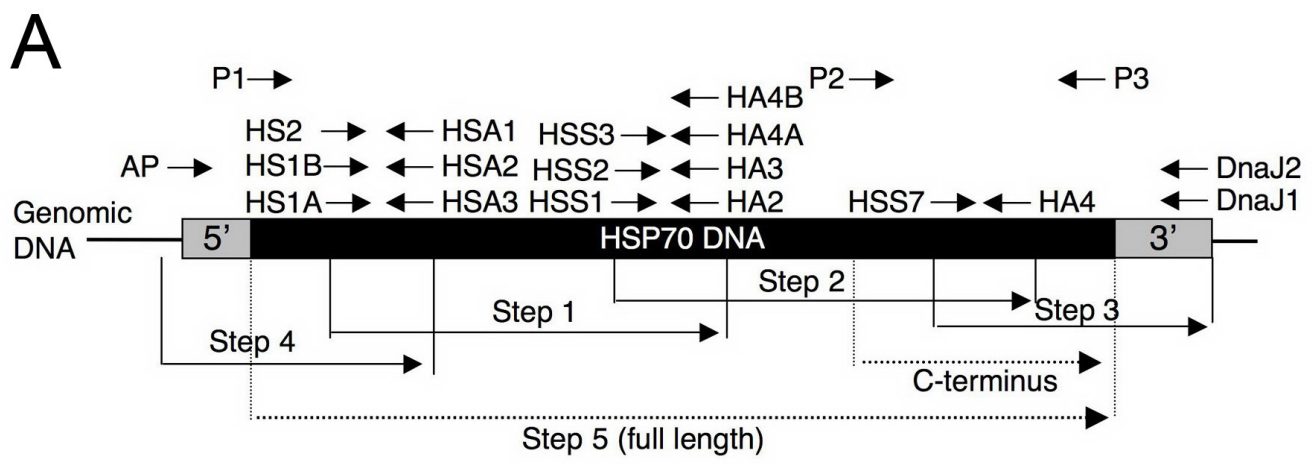

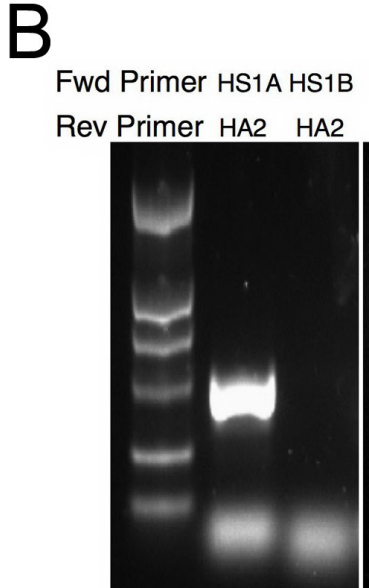

Step 1

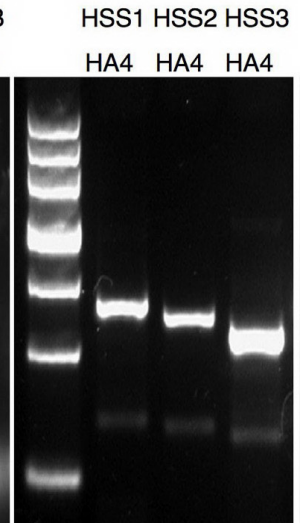

Step 2

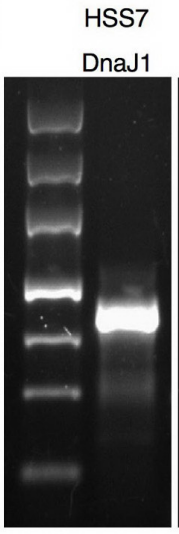

Step 3

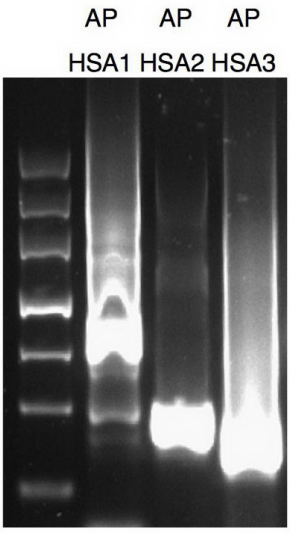

Step 4

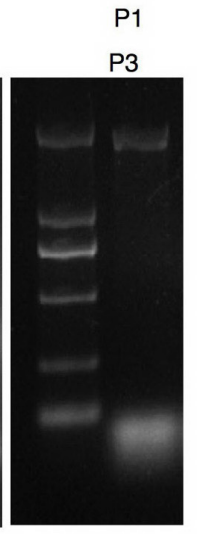

Step 5

Figure 1. Cloning of Mycoplasma ovipneumoniae HSP70 DNA. A. Schematic diagram illustrating the procedure and strategy of M. ovipneumoniae HSP70 DNA cloning. The procedure was conducted step by step as described in the Material and Methods section. B. Ethidium bromide agarose gel images of PCR products of each step (bottom) using the indicated primer set(s) (top). A DNA ladder was loaded in the left lane of each gel.

\section{Bioinformatic analysis of the M. ovipneumoniae HSP70 gene}

The ORF of the M. ovipneumoniae HSP70 gene was determined by inputting the identified sequence and the homology with other Mycoplasma HSP70 proteins using the NCBI ORF finder software. The full-length gene is 1812 in length encoding 604 amino acids with a predicted molecular mass of $66.1 \mathrm{kDa}$ (Figure 2). The codon usage in the coding region of HSP70 had a strong preference for A or T at the third position. Similar to mitochondrial and other mycoplasma genomes, tryptophan was encoded by the UGA codon. This was consistent with previous findings (Chou et al., 1997; Falah and Gupta, 1997). The molecular mass of the M. ovipneumoniae HSP70 gene is $1098.5 \mathrm{kDa}$. The $\mathrm{G} / \mathrm{C}$ content was $34.16 \%$, which is higher than the $28 \%$ average seen in mycoplasmal genomic DNA (Razin et al., 1998). Comparative DNA and amino acid sequence analysis between 16 mycoplasmal HSP70 genes revealed that 


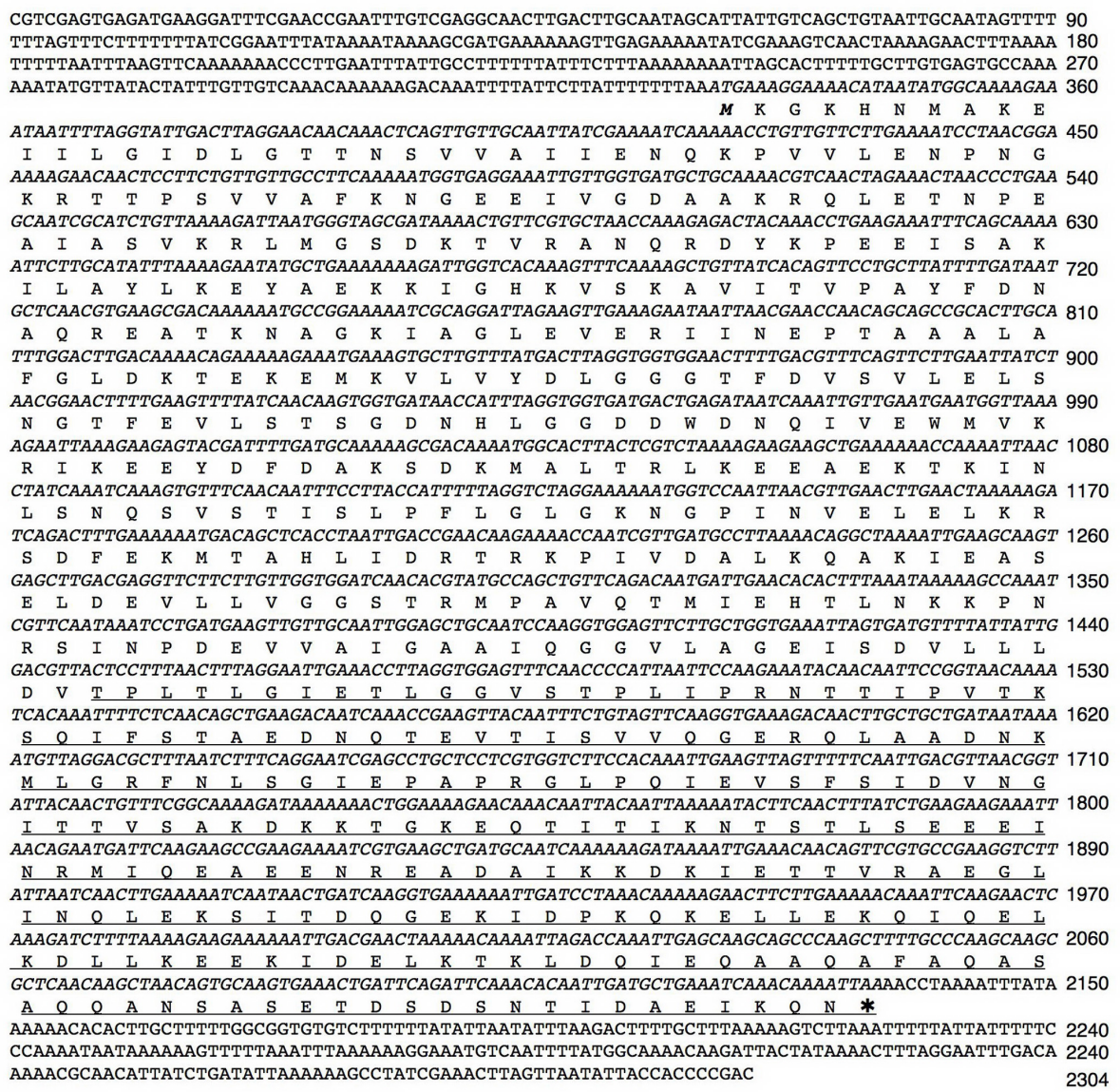

Figure 2. DNA sequence and predicted amino acid sequence of Mycoplasma ovipneumoniae HSP70. The italic letters represent HSP70 coding sequences. The bold italic $M$ shows the predicted start methionine of the ORF, with an asterisk ${ }^{*}$ ) indicating the stop codon. The underlined amino acid sequences indicate the COOH-terminus of the HSP70 gene that was cloned into the Escherichia coli expression vector pET-28a $(+)$ for generation of recombinant protein.

the M. ovipneumoniae had an identity between 59 to $87 \%$ and 58 to $94 \%$, respectively. Furthermore, M. ovipneumoniae and $M$. hyo shared the highest DNA and amino acid sequence identity of 87 and $94 \%$, respectively (Table 2). To understand the genetic relationship between M. ovipneumoniae HSP70 and other Mycoplasma HSP70s, phylogenic trees were produced using the nucleotide and amino acid sequences (Figure 3). Phylogenetic analysis revealed that Mycoplasma HSP70 was divided into two major branches. The first branch included $M$. ovipneumoniae and $M$. hyo. The other branch was more diverse including M. capricolum, $M$. pneumoniae and M. genitalium. These two branches contained several sub-branches (Figure $3)$. Both the DNA and amino acid identities to other mycoplasmal HSP70 genes decreased according to the degree of phylogenetic relationship between Mycoplasma species (strains), and exhibited the lowest sequence identity of both DNA and amino acid with M. pneumoniae, which were 58 and 59\%, respectively (Table 2 and Figure 3). 
A

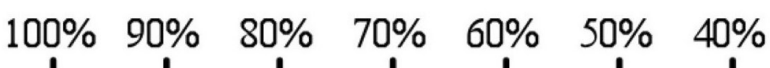

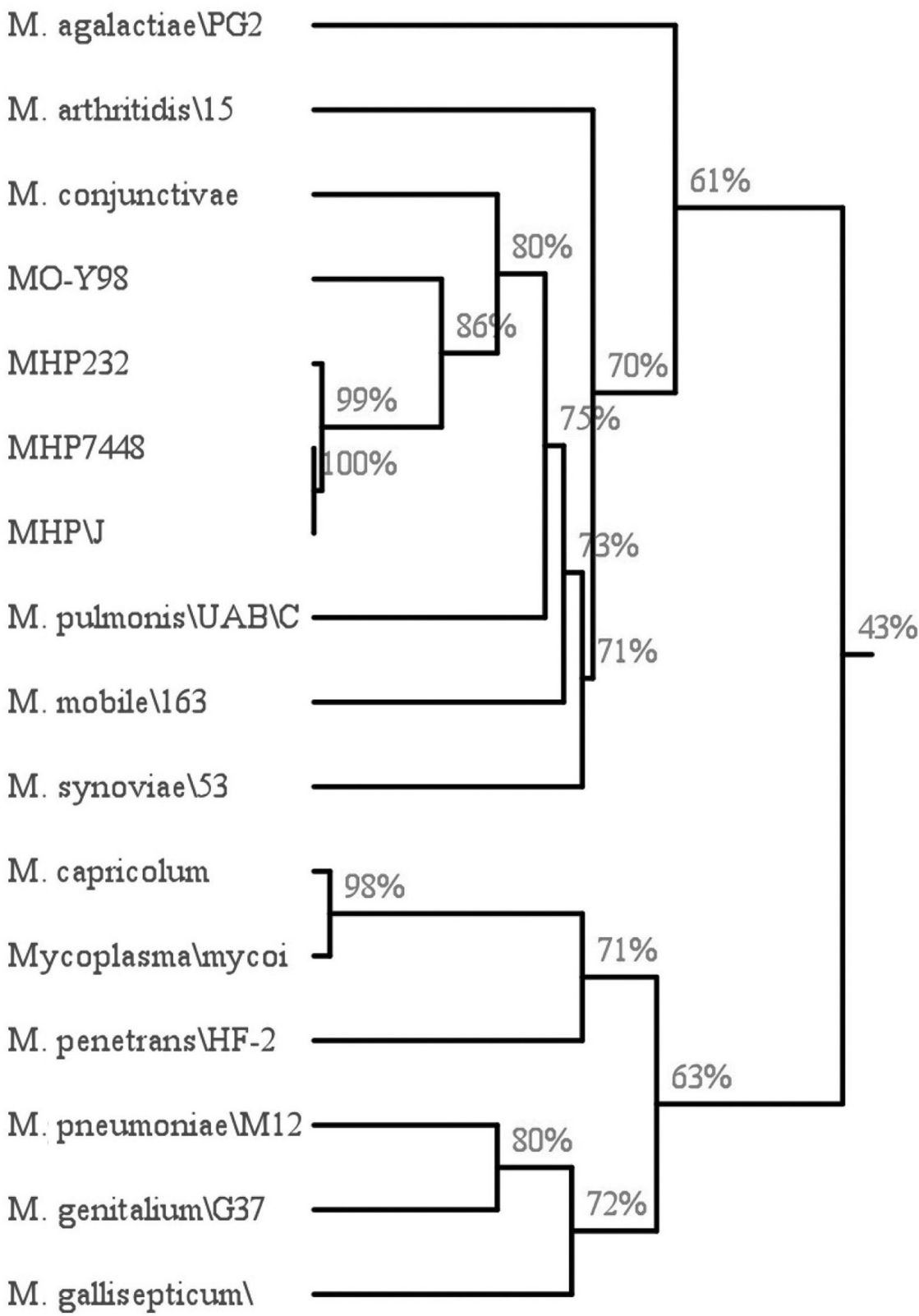

Figure 3. Continued on next page. 

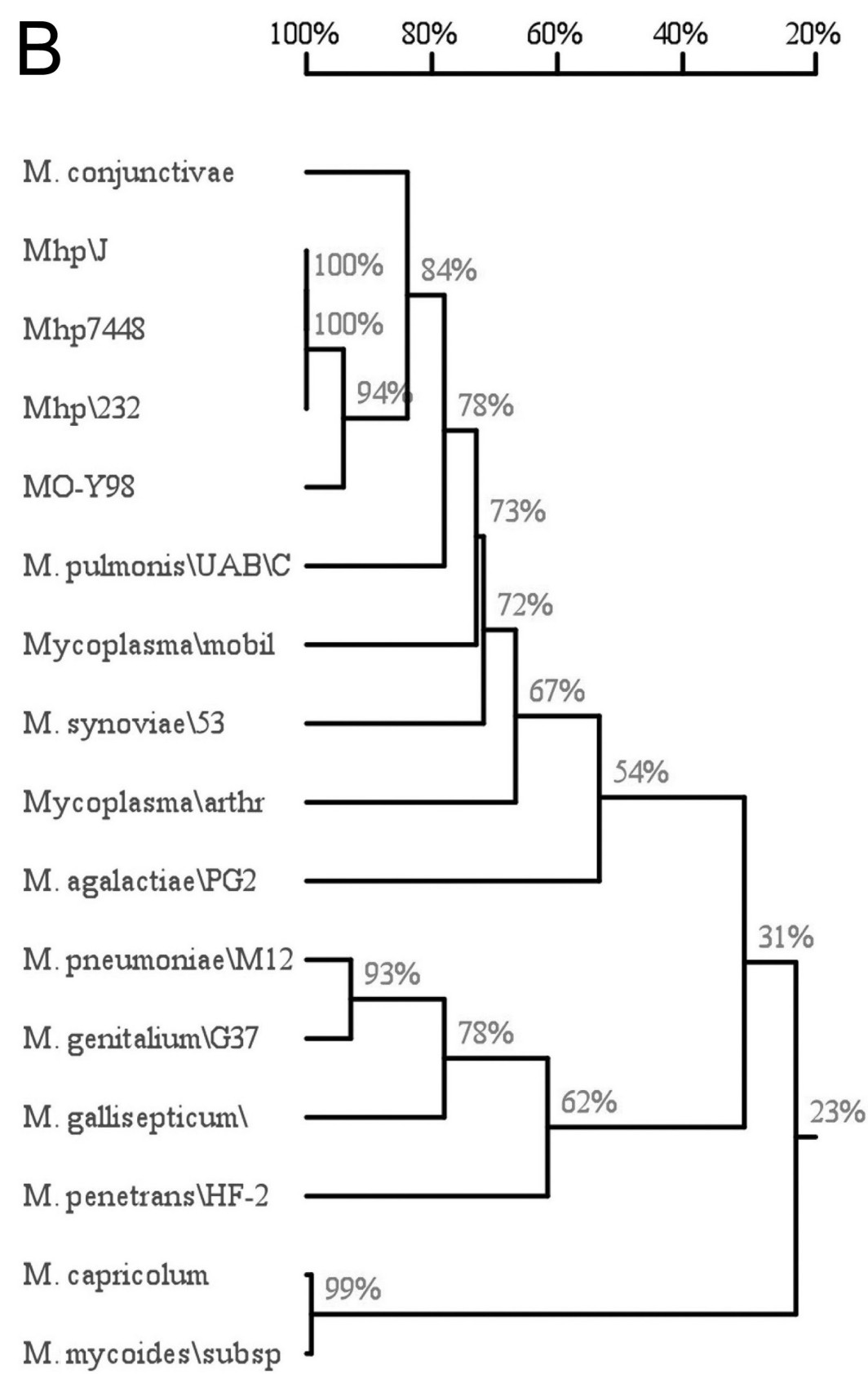

Figure 3. Dendrogram of the phylogenetic relationship. The phylogenetic relationship between the HSP70 DNA(A) and protein sequences (B) of 16 different Mycoplasma species (strains) was based on a neighbor-joining algorithm. The trees were constructed using the p-distance method; bootstrap values are shown next to the branches. The size of each branch is proportional to the evolutionary distances used to generate the phylogenetic tree. The accession numbers of Mycoplasma HSP70 were obtained from the NCBI database. 


\section{Expression of the recombinant C-terminus of the M. ovipneumoniae HSP70 protein}

The carboxy-terminal portion of HSP70 has been suggested as the major target for the humoral immune response (Kakeya et al., 1999). A bacterial expression vector, pET-MoHsp70C, capable of expressing the C-terminal portion of M. ovipneumoniae HSP70, was generated to evaluate its potential immunogenic function. E. coli BL21 (DE3) cells were transformed with pET-MoHsp70C and incubated in LB media containing appropriate antibiotics for 18-24 h. This was followed by a 4-h $1.0 \mathrm{mM}$ IPTG induction. SDS-PAGE analysis of whole cell lysate demonstrated a $\sim 29-\mathrm{kDa}$ target band corresponding to the expected band (left Panel in Figure 4A). A 6X His-tag fused to the C-terminal portion of the target proteins is achieved by expressing proteins using a pET vector. The integrity and identity of the fusion protein may be ascertained by His-tag detection by Western blot analysis. Blotting for this tag demonstrated a single 29-kDa protein band (right Panel in Figure 4A) representing the recombinant C-terminal portion of HSP70.
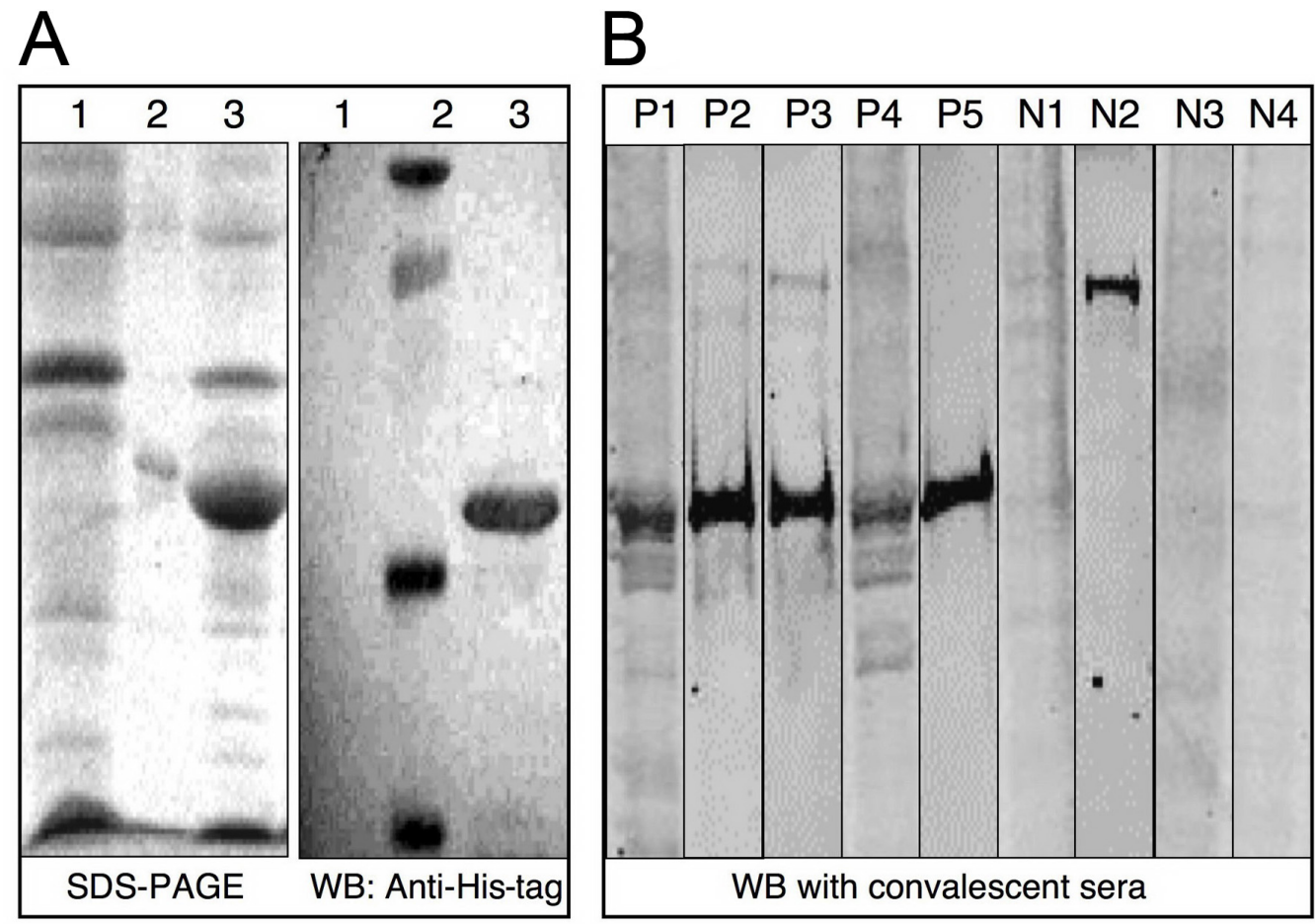

Figure 4. Expression and immunoblotting analysis of recombinant HSP70 protein. A. Western blotting analysis of the expression of recombinant C-terminus HSP70 of Mycoplasma ovipneumoniae. The left panel shows the SDSPAGE result of cell lysate derived from Escherichia coli BL21 (DE3) transformed with pET-28a (Lane 1), protein molecular weight markers (Lane 2) and pET-MoHSP70C with IPTG induction (Lane 3). The right panel displays the resulting Western blot (WB) of recombinant fusion protein detected with anti-His antibody. B. Immunoblotting analysis of the recombinant HSP70 fusion protein using ELISA-positive sheep convalescent sera (P1-P5 were sera from 5 individual sheep) and ELISA-negative sheep sera (N1-N4 were sera from 4 individual sheep). 


\section{Antibody reaction of convalescent sera with the recombinant $\mathrm{C}$-terminal portion of the M. ovipneumoniae HSP70 protein}

Western blot analysis was used to verify whether HSP70 expression induces an immune response by using antibodies generated against HSP70 to screen convalescent sera derived from $M$. ovipneumoniae-infected sheep. The recombinant $\mathrm{C}$-terminal portion of the $M$. ovipneumoniae HSP70 protein was detected by Western immunoblot using ELISA-positive sheep convalescent sera, but not in the ELISA-negative sera (Figure 4B). This result suggests that the HSP70 may be a dominant immunogen that induces the host immune response against mycoplasmal infection. Furthermore, recombinant HSP70 may be a potential vaccine candidate and/or vaccine adjuvant against $M$. ovipneumoniae infections.

\section{DISCUSSION}

A number of studies have demonstrated that HSPs play critical roles in both the innate and adaptive immunity functioning as immune adjuvant and/or immunogens (Craig, 1985). HSP70 has been suggested to be involved in the antigen processing and presentation machinery associated with a transporter related to antigen processing and proteasomes that degrade cellular proteins to produce antigen peptides (Torigoe et al., 2009). M. tuberculosis HSP70 has been shown to be a strong antigen containing multiple B- and T-cell epitopes. Furthermore, it has been demonstrated that the antigenic properties can be exploited to enhance and induce the humoral and cellular immune responses as both an adjuvant and immunogen (Suzue and Young, 1996; Li et al., 2006). A previous study has identified a 42-kDa recombinant $M$. hyopneumoniae HSP70 protein by an immunoscreening assay with porcine convalescent and hyperimmune sera. This part of $M$. hyopneumoniae HSP70 was sequence homologous to that of M. genitalium and B. subtilis. Evidence that purified monospecific antibodies to a portion of HSP70 was capable of inhibiting the growth of M. hyopneumoniae suggested the potential use of HSP70 as a vaccine (Chou et al., 1997). M. ovipneumoniae and M. hyopneumoniae are members of the order Mycoplasmales and were the sequences with the highest homology (Minion et al., 2004). These studies implied an importance of HSP70 in host immune responses against $M$. ovipneumoniae infection. However, neither the entire genome nor the HSP70 gene of M. ovipneumoniae has been sequenced.

In this report, we described the cloning and phylogenetic analysis of the M. ovipneumoniae HSP70/DnaK gene. The M. ovipneumoniae HSP70 gene encompasses 1812 proteinencoding sequences and is located downstream of DnaJ. Previous phylogenetic analysis of HSP70 has demonstrated that Mycoplasma species are closely related to Gram-positive bacteria with evidence of low G/C content in all 16 Mycoplasma HSP70 sequences analyzed (Falah and Gupta, 1994, 1997). In the present study, the analysis of DNA and predicted amino acid sequences of Mycoplasma HSP70 revealed a high degree of identity between the HSP70 gene of M. ovipneumoniae and M. hyopneumoniae, the identity of the HSP70 sequence decreased with the degree of phylogenetic relationship between Mycoplasma species.

HSP70 is a major antigen of pathogenic bacteria and mycoplasmas, such as M. tuberculosis and M. hyopneumoniae (Suzue and Young, 1996; Chou et al., 1997; Li et al., 2006). The predicted tertiary structure of M. ovipneumoniae HSP70 revealed that the C-terminus of the protein was outside of the tertiary structure, implying that an antigenic epitope against $M$. 
ovipneumoniae infection may exist in the C-terminus of the HSP70 protein. To investigate the antigenicity of HSP70 in M. ovipneumoniae infections, the C-terminal portion of the HSP70 gene was cloned into a bacterial expression vector and expressed in E. coli cells. The bacterial recombinant protein was used to detect specific antibodies against the HSP70 protein in sheep convalescent sera. As demonstrated with M. hyopneumoniae HSP70 (Chou et al., 1997), the immunoblotting assay demonstrated that the recombinant protein strongly reacted with the ELISA-positive sera, with only weak or no reaction to the negative sera. This suggests that $M$. ovipneumoniae HSP70 protein may be a relevant antigen for vaccine development against $M$. ovipneumoniae infections.

In conclusion, this study described the cloning and characterization of the $M$. ovipneumoniae HSP70 gene. Comparative analysis of 16 Mycoplasma HSP70 genes demonstrated that the HSP70 shared the highest sequence identity with M. hyopneumoniae HSP70. The evidence that the recombinant C-terminal portion of the HSP70 protein enables it to react with convalescent sera from M. ovipneumoniae-infected sheep suggested that HSP70 may be a relevant antigen for vaccine development.

\section{Conflict of interest}

The authors declare that there are no conflicts of interest.

\section{ACKNOWLEDGMENTS}

Research supported by a sub-project of the National Basic Research Program of China (\#2006CB504401), the National Natural Science Foundation of China (\#30860207, \#30960289), the Scientific and technological projects of Ningxia Hui Autonomous Region (\#Z2006-1-75001, \#KGZ-12-10-02), Grant of Science and Technology Program of Ningxia to Y.J. Wang and the Key Science and Technology Research Projects of Colleges and Universities of Ningxia Hui Autonomous Region.

\section{REFERENCES}

Amemiya K, Meyers JL, Deshazer D, Riggins RN, et al. (2007). Detection of the host immune response to Burkholderia mallei heat-shock proteins GroEL and DnaK in a glanders patient and infected mice. Diagn. Microbiol. Infect. Dis. 59: 137-147.

Barré A, de Daruvar A and Blanchard A (2004). MolliGen, a database dedicated to the comparative genomics of Mollicutes. Nucleic Acids Res. 32: D307-D310.

Biberfeld G (1985). Infection Sequelae and Autoimmune Reactions in Mycoplasma pneumoniae Infection. Academic Press, Inc., Orlando, 293-311.

Candiano G, Bruschi M, Musante L, Santucci L, et al. (2004). Blue silver: a very sensitive colloidal Coomassie G-250 staining for proteome analysis. Electrophoresis 25: 1327-1333.

Chou SY, Chung TL, Chen RJ, Ro LH, et al. (1997). Molecular cloning and analysis of a HSP (heat shock protein)-like 42 kDa antigen gene of Mycoplasma hyopneumoniae. Biochem. Mol. Biol. Int. 41: 821-831.

Craig EA (1985). The heat shock response. CRC Crit. Rev. Biochem. 18: 239-280.

Craig EA, Gambill BD and Nelson RJ (1993). Heat shock proteins: molecular chaperones of protein biogenesis. Microbiol. Rev. 57: 402-414.

Dassanayake RP, Shanthalingam S, Herndon CN, Subramaniam R, et al. (2010). Mycoplasma ovipneumoniae can predispose bighorn sheep to fatal Mannheimia haemolytica pneumonia. Vet. Microbiol. 145: 354-359.

Falah M and Gupta RS (1994). Cloning of the hsp70 (dnaK) genes from Rhizobium meliloti and Pseudomonas cepacia: 
phylogenetic analyses of mitochondrial origin based on a highly conserved protein sequence. J. Bacteriol. 176: 7748-7753.

Falah M and Gupta RS (1997). Phylogenetic analysis of mycoplasmas based on Hsp70 sequences: cloning of the dnaK (hsp70) gene region of Mycoplasma capricolum. Int. J. Syst. Bacteriol. 47: 38-45.

Floto RA, MacAry PA, Boname JM, Mien TS, et al. (2006). Dendritic cell stimulation by mycobacterial Hsp70 is mediated through CCR5. Science 314: 454-458.

Foggie A, Jones GE and Buxton D (1976). The experimental infection of specific pathogen free lambs with Mycoplasma ovipneumoniae. Res. Vet. Sci. 21: 28-35.

Fraser CM, Gocayne JD, White O, Adams MD, et al. (1995). The minimal gene complement of Mycoplasma genitalium. Science 270: 397-403.

Himmelreich R, Hilbert H, Plagens H, Pirkl E, et al. (1996). Complete sequence analysis of the genome of the bacterium Mycoplasma pneumoniae. Nucleic Acids Res. 24: 4420-4449.

Ionas G, Clarke JK and Marshall RB (1991). The isolation of multiple strains of Mycoplasma ovipneumoniae from individual pneumonic sheep lungs. Vet. Microbiol. 29: 349-360.

Jones GE, Foggie A, Mould DL and Livitt S (1976). The comparison and characterisation of glycolytic mycoplasmas isolated from the respiratory tract of sheep. J. Med. Microbiol. 9: 39-52.

Kakeya H, Udono H, Maesaki S, Sasaki E, et al. (1999). Heat shock protein 70 (hsp70) as a major target of the antibody response in patients with pulmonary cryptococcosis. Clin. Exp. Immunol. 115: 485-490.

Kiang JG and Tsokos GC (1998). Heat shock protein 70 kDa: molecular biology, biochemistry, and physiology. Pharmacol. Ther. 80: 183-201.

Kumar S, Tamura K and Nei M (2004). MEGA3: integrated software for molecular evolutionary genetics analysis and sequence alignment. Brief. Bioinform. 5: 150-163.

Li X, Yang X, Li L, Liu H, et al. (2006). A truncated C-terminal fragment of Mycobacterium tuberculosis HSP70 gene enhanced potency of HBV DNA vaccine. Vaccine 24: 3321-3331.

Lin YC, Miles RJ, Nicholas RA, Kelly DP, et al. (2008). Isolation and immunological detection of Mycoplasma ovipneumoniae in sheep with atypical pneumonia, and lack of a role for Mycoplasma arginini. Res. Vet. Sci. 84: 367-373.

Minion FC, Lefkowitz EJ, Madsen ML, Cleary BJ, et al. (2004). The genome sequence of Mycoplasma hyopneumoniae strain 232, the agent of swine mycoplasmosis. J. Bacteriol. 186: 7123-7133.

Ramagli L (1999). 2-D Proteome Analysis. In: Methods in Molecular Biology (Link A, ed.). Humana Press, Totowa, 99-103.

Rasoli M, Omar AR, Aini I, Jalilian B, et al. (2010). Fusion of HSP70 gene of Mycobacterium tuberculosis to hemagglutinin (H5) gene of avian influenza virus in DNA vaccine enhances its potency. Acta Virol. 54: 33-39.

Razin S, Yogev D and Naot Y (1998). Molecular biology and pathogenicity of mycoplasmas. Microbiol. Mol. Biol. Rev. 62: 1094-1156.

Saitou N and Nei M (1987). The neighbor-joining method: a new method for reconstructing phylogenetic trees. Mol. Biol. Evol. 4: 406-425.

Scherm B, Gerlach GF and Runge M (2002). Analysis of heat shock protein 60 encoding genes of mycoplasmas and investigations concerning their role in immunity and infection. Vet. Microbiol. 89: 141-150.

Staint George TD and Carmichael LE (1975). Isolation of Mycoplasma ovipneumoniae from sheep with chronic pneumonia. Vet. Rec. 97: 205-206.

Suzue K and Young RA (1996). Adjuvant-free hsp70 fusion protein system elicits humoral and cellular immune responses to HIV-1 p24. J. Immunol. 156: 873-879.

Torigoe T, Tamura Y and Sato N (2009). Heat shock proteins and immunity: application of hyperthermia for immunomodulation. Int. J. Hyperther. 25: 610-616. 